\section{Destruction of Thyroxine by Electrolytic Desalting}

Ir is well known that artefacts and serious distortion can be caused during paper chromatography if acids are present in the solution being chromatographed, and that they can be eliminated by initial electrolytic desalting. In a recent communication ${ }^{1}$, Acland confirmed that trichloracetic acid can be "largely" removed by desalting the solution electrolytically ; as an example, he showed that the chromatographic picture from a mixture of thyroxine and trichloracetic acid was very distorted, whereas that from a mixture of thyroxine and 'desalted' trichloracetic acid was relatively undistorted.

It might therefore be supposed that electrolytic desalting of thyroxine extracts containing trichloracetic acid would improve their chromatographic investigation. We wish to point out that this is not the case, as the electrolytic desalting of thyroxine solutions leads to rapid reductive deiodination. After $10 \mathrm{mgm}$. of thyroxine, dissolved in $1 \mathrm{ml}$. of aqueous methanol containing trichloracetic acid or any other acid, had been subjected to electrolytic desalting at 500-250 m.amp. for $10 \mathrm{~min}$., paper chromatography showed only thyronine to be present (ninhydrin reagent), with no detectable organically bound iodine (ceric sulphate - arsenite reagent, which would have shown as little as 1 per cent remaining ${ }^{2}$ ). Desalting for shorter periods $(1 \mathrm{~min}$.) gave intermediate products identified as various iodo-thyronines. When an aqueous solution of trichloracetic acid ( $1 \mathrm{ml}$. containing $10 \mathrm{mgm}$.) was desalted at 500 $100 \mathrm{~m}$.amp. for $7 \mathrm{~min}$. its $p \mathrm{H}$ rose to $5 \cdot 5$, indicating virtually complete removal of the acid.

Further work on the stepwise dehalogenation of polyhalogen derivatives of tyrosine and thyronine will be reported elsewhere ${ }^{3}$.

\section{J. B. JEPSON}

I. SMITH

Courtauld Institute of Biochemistry,

Middlesex Hospital Medical School, London, W.1. Nor. 1.

1 Acland, J. D., Nature, 176, 694 (1955).

${ }^{2}$ Bowden, C. H., Maclagen, N. F., and Wilkinson, J. H., Biochem. J., $59,93(1955)$.

${ }^{s}$ Smith, I., Stevens, B. J., and Jepson, J. B., Proc. Biochem. Soc, November meeting, Biochem. J. (in the press).

\section{Use of Gas - Liquid Partition Chromatography as a Preparative Method}

The sharp separations obtainable by gas-liquid partition chomatography ${ }^{1}$ would make it a useful alternative to distillation as a method for the purifica. tion of volatile compounds if the quality of the separations could be maintained on large columns. Evans and Tatlow ${ }^{2}$ have described a column, $16 \mathrm{ft}$. long by $3 \mathrm{~cm}$. internal diameter, which is suitable for the separation of $2-8 \mathrm{gm}$. of fluorocarbons. It is not yet clear, however, how much further the increase in size can be carried; but in any event, as so far developed, chromatography remains a batch process. Hence another line of approach to the problem of processing large amounts of material would be the autornatic repetition of the cycle of batch operations, that is, injection of the charge and isolation of the pure material as it emerges from the column. This approsch would be applicable whatever size columns are finally adopted.

Under fixed column conditions, each substance emerges at a constant time after injection, and this suggested that a clock might be used for controlling the cyclic process. We have constructed such a clock and used it successfully in the separation of $\mathrm{C}_{4}$ hydrocarbon gases. In itself, the chromatographic apparatus is similar to those previously described, except that the column is somewhat larger $(1 \mathrm{~m}$. long by 15. $\mathrm{mm}$. internal diameter) and two solenoid-operated valves are incorporated in the flow line. The gaseous sample is admitted to the column through one valve and the outlet gas can be deflected from one to another of two collecting traps by means of the other valve, which is located immediately. after the detector'. The clock comprises a 'Londex' $P R / S$ timer and a 50-position uniselector arranged to give a cycle of variable length divided into fifty equal intervals. At the beginning of each cycle, the inlet valve opens and a charge of the hydrocarbon gas is admitted to the column inlet, the amount depending on the pressure and the length of time the valve is open. 'The recorder trace for the detector is observed, and those positions of the uniselector corresponding to the peak required are wired together to operate the outlet valve and divert the flow of gas; in this way the wanted material is separated from the remainder. At a given column temperature the times of emergence of the component peaks can be varied by altering the rate of flow of the carrier gas which, together with the length of cycle, is arranged so that all the remaining components are eluted from the column before the next appearance of the peak required. So far, we have used cycles of $15-20 \mathrm{~min}$. duration, (nitrogen) carrier-gas rates of about $30 \mathrm{l} / \mathrm{hr}$. and a charge of somewhat less than $1 \mathrm{gm}$. (giving a daily throughput of between 70 and $80 \mathrm{gm}$.). Lowboiling materials prove difficult to remove from the carrier-gas stream, and it has been found most satisfactory to fill the collecting traps with activated alumina gel. In use they are immersed in a Dewar vessel containing solid carbon dioxide, and the gas can easily be recovered on warming.

The success of the method depends upon the pattern produced on the recorder remaining in phase with the clock. Such constancy in the times of emergence of the components of a mixture has been achieved in the trials so far carried out by attention to three factors : the temperature of the column (which was packed on ice), the rate of flow of the carrier gas, and its presaturation with the solvent used. In these conditions successful runs, lasting several days, have resulted in the expected separation and no adjust. ments to the clock have been required.

The work described has been carried out as part of the programme of the Chemical Research Laboratory, by permission of the Director of which this communication is published.

\section{Ambrose \\ R. R. COLLERSON}

Chemical Research Laboratory,

Teddington, Middlesex. Oct. 28. ${ }^{1}$ James, A. T., and Martin, A. J. P., Biochem. J., 50, 679 (1952), 41, 80 (1955).

2 Evans, D. E. M., and Tatlow, J. C., J. Chem. Soc., 1184 (1955).

${ }^{3}$ Ambrose, D., and Collerson, R. R., J. Sci. Instr., 32, 323 (1955). 